\title{
On the Discontinuity of the Costates for Optimal Control Problems With Coloumb Friction
}

\author{
Brian J. Driessen \\ Sandia National Laboratories, Albuquerque, NM 87185-0847, bjdries@ sandia.gov \\ Nader Sadegh \\ Georgia Institute of Technology, Atlanta, GA 30332, nader.sadegh@me.gatech.edu
}

\begin{abstract}
This work points out that the costates are actually discontinuous functions of time for optimal control problems with Coloumb friction. In particular these discontinuities occur at the time points where the velocity of the system changes sign. To our knowledge, this has not been noted before. This phenomenon is demonstrated on a minimum-time problem with Coloumb friction and the consistency of discontinuous costates and switching functions with respect to the input switches is shown.
\end{abstract}

\section{Introduction}

Most optimal control research work has dealt with systems in which the state equation is continuous in the states. Exceptions include the work of [Willigenburg and Loop, 1991] who included Coloumb friction when generating minimum-time trajectories of a rigid two-link robotic arm. Since the costates for such a problem are discontinuous, as we shall see, the switching functions should also be discontinuous. However, no such discontinuities could be seen in the plots given in [Willigenburg and Loop, 1991]. It is conceivable that the discrepancies between the input switches and the switching functions observed by these researchers could be explained by the fact that they did not include the "jumps" or discontinuities in the costates.

Herein we will explain why one might expect discontinuities in the costates, and we will demonstrate that the calculated discontinuities yield consistency between the switching functions and the input switches for a minimum-time problem.

\section{Motivation for Discontinuous Costates}

The reason to expect discontinuities in the costates is best explained with a simple example. Consider the following optimal control problem.

$$
\operatorname{Min}_{f}
$$

subject to

$$
\begin{aligned}
& \ddot{q}=u-\operatorname{sign}(\dot{q}) \\
& q(0)=q_{0} \\
& \dot{q}(0)=v_{0}<0 \\
& q\left(t_{f}\right)=q_{f}>q_{0} \\
& \dot{q}\left(t_{f}\right)=0 \\
& -u_{\max } \leq u \leq u_{\max }>1
\end{aligned}
$$

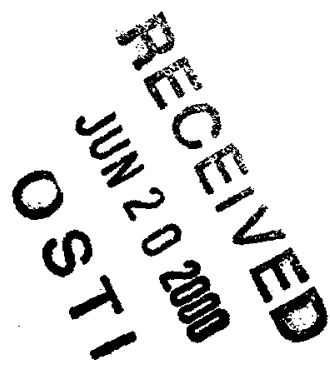

Letting $x_{1}=q$ and $x_{2}=\dot{q}$, the state equation is

$$
\left(\begin{array}{c}
\dot{x}_{1} \\
\dot{x}_{2}
\end{array}\right)=\left(\begin{array}{c}
x_{2} \\
u-\operatorname{sign}\left(x_{2}\right)
\end{array}\right)
$$

The Hamiltonian is

$$
H=1+\lambda_{1} x_{2}+\lambda_{2} u-\lambda_{2} \operatorname{sign}\left(x_{2}\right)
$$

where $\lambda_{1}$ and $\lambda_{2}$ are the costates. Then,

$$
\frac{\partial H}{\partial x_{2}}=\lambda_{1}-2 \lambda_{2} \delta\left(x_{2}\right)
$$

where $\delta(\cdot)$ is the dirac delta function, which appears because of the infinite derivative of the signum function. The factor of 2 in (10) appears because the magnitude of

\footnotetext{
${ }^{1}$ Sandia is a multiprogram laboratory operated by Sandia Corporation, a Lockheed Martin Company, for the United States Department of Energy under Contract DE-AC04-94AL85000.
} 


\section{DISCLAIMER}

This report was prepared as an account of work sponsored by an agency of the United States Government. Neither the United States Government nor any agency thereof, nor any of their employees, make any warranty, express or implied, or assumes any legal liability or responsibility for the accuracy, completeness, or usefulness of any information, apparatus, product, or process disclosed, or represents that its use would not infringe privately owned rights. Reference herein to any specific commercial product, process, or service by trade name, trademark, manufacturer, or otherwise does not necessarily constitute or imply its endorsement, recommendation, or favoring by the United States Government or any agency thereof. The views and opinions of authors expressed herein do not necessarily state or reflect those of the United States Government or any agency thereof. 


\section{DISCLAIMER}

Portions of this document may be illegible in electronic image products. Images are produced from the best available original document. 
the instantaneous change in the signum function is 2 . Since

$$
\dot{\lambda}=-\frac{\partial H}{\partial x}
$$

we have

$$
\begin{aligned}
& \dot{\lambda}_{1}=0 \\
& \dot{\lambda}_{2}=-\lambda_{1}+2 \lambda_{2} \delta\left(x_{2}\right)
\end{aligned}
$$

\section{Numerical Demonstration of Consistency of Discontinuous Costates}

In this section we will solve the optimal control problem of section 2. Consider the case of $u_{\max }=2$, $v_{0}=-1$, and $q_{f}=1$. Figure 1 below shows the minimumtime input, states, and costates.

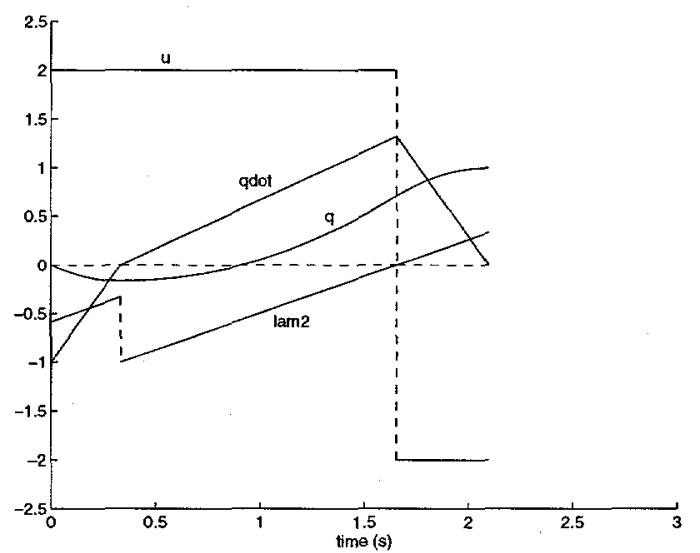

Figure 1. Input, States, and Costates Versus Time

We see in Figure 1 that the input is bang-bang, switching from its maximum value to its minimum value. Let $t_{1}$ be the time the velocity $\dot{q}$ changes sign and $t_{s}$ the time the input switches. From time zero to the time $t_{1}=0.3333 \mathrm{~s}$ at which the velocity changes sign, the acceleration is at its maximum possible magnitude of $\left|-u_{\max }-1\right|=|-3|$, where the friction force actually helps the input bring the velocity to zero.

From $t_{1}$ to the input switch time $t_{s}=1.656 \mathrm{~s}$, the acceleration is only $u_{\max }-1=1$, where the friction force is now opposing the input. Finally, after $t_{s}$, the friction force aids the input again in bringing the velocity to zero at the final time $t_{f}$.

Using the fact that the value of $\lambda(0)$ is given by the derivative of the cost-to-go with the initial state, one can calculate $\lambda(0)$ to be:

$$
\lambda(0)=\left(\begin{array}{l}
-0.7559 \\
-0.5853
\end{array}\right)
$$

Hence, we have from (13):

$$
\dot{\lambda}_{2}=0.7559+2 \lambda_{2} \delta\left(t-t_{1}\right)
$$

$$
\lambda_{2}(0)=-0.5853
$$

The value of $\lambda_{2}\left(t_{1}{ }^{-}\right)$just before the velocity changes sign is from (15) and (16):

$$
\lambda_{2}\left(t_{1}^{-}\right)=-1 / 3
$$

which is half the value of the jump in $\lambda_{2}$. In Figure 1, we observe this discontinuity in $\lambda_{2}$. Clearly, without this jump in $\lambda_{2}, \lambda_{2}$ would not be zero at the switching time $t_{s}$.

\section{Conclusion}

We demonstrated that the costates and switching functions are not continuous functions of time for problems with Coloumb friction, because the derivative of the signum function is $2 \delta\left(t-t_{1}\right)$ where $t_{1}$ is the time at which the argument of the signum funrction changes sign. This may explain discrepancies observed in previous work in which switching function appeared to have been calculated to be continuous despite the presence of Coloumb friction and changes in the velocity's sign.

\section{References}

[1] Willigenbug, L. and Loop, R., "Computation of Time-Optimal Controls Applied to Rigid Manipulators With Friction," International Journal of Control, Vol. 54, 1991, pp. 1097-1117.

and 